\title{
NEAR IR - OBSERVATIONS OF THE JET IN M87
}

\author{
M. NEUMANN, K. MEISENHEIMER, H.-J. RÖSER and M. STICKEL \\ Max Planck - Institut für Astronomie, \\ Königstuhl 17, D - 69117 Heidelberg
}

The central region of M87 in the $\mathrm{K}^{\prime}(2.1 \mu \mathrm{m})$ - band was imaged using the $2.2 \mathrm{~m}$ telescope on Calar Alto, Spain. In addition to the jet our $\mathrm{K}^{\prime}$ exposure (Fig. 1) shows an arclike structure $\sim 24$ arcsec southeast of the nucleus. This is the IR counterpart to the feature detected by Stiavelli et al. (1992) in the optical and to the brightness maximum of the southeastern radiolobe designated as "theta" (Hines et al. 1989). Its radio to IR spectral index is $\alpha=-0.88 \pm 0.01\left(S_{\nu} \propto \nu^{\alpha}\right)$, consistent with the radio spectral index of $\alpha=-0.84 \pm 0.07$ (Salter et al. 1989) of the whole radio lobe measured between $5 \mathrm{GHz}$ and $230 \mathrm{GHz}$.

A second object can possibly be assigned to a bright radio feature at the southern limb of the eastern radio lobe called "eta" by Hines et al. (1989). This apparent association will be investigated with further IR observations of higher spatial resolution and higher SNR.

A detailed photometric analysis of the jet will be presented elsewhere.

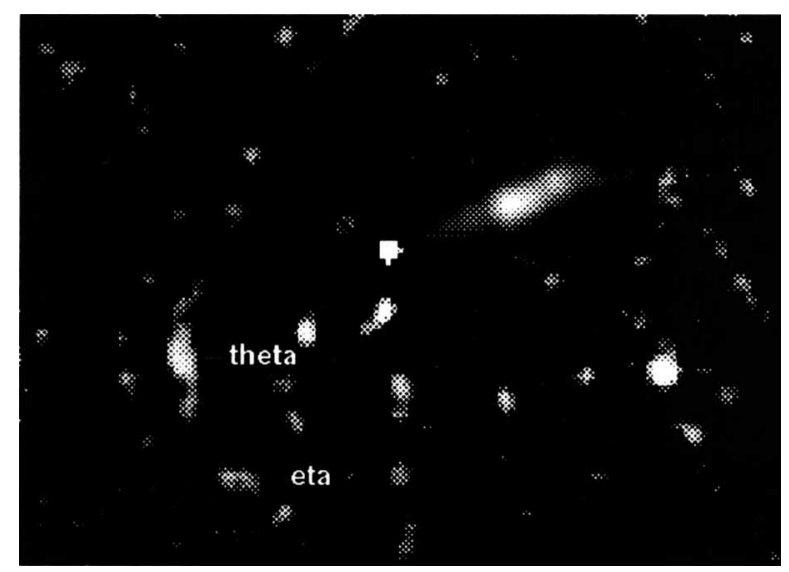

Fig. 1. 540 sec K' - exposure of M87 after subtraction of a smooth galaxy model. North is up and east to the left. The jet has a length of $\sim 20$ arcsec. Intensity range is $2 \sigma \ldots 507 \sigma$ for the jet and $2 \sigma \ldots 39 \sigma$ for all other features including theta.

\section{References}

Hines, D.C., Owen, F.N., Eilek, J.A., 1989, ApJ 347, 713

Salter, C.J., Chini, R., Haslam, C.G.T., Junor, W., Kreysa, E., Mezger, P.G., Wink, J.E., Zylka, R., 1989, A\&A 220, 42

Stiavelli, M., Biretta, J., Møller, P., Zeilinger, W.W., 1992, Nature 355, 802

329

T. J.-L. Courvoisier and A. Blecha: Multi-Wavelength Continuum Emission of AGN, 329.

C 1994 IAU. Printed in the Netherlands. 